\title{
Resonant modes and magnetoelectric performance of PZT/Ni cylindrical layered composites
}

\author{
D.A. Pan · S.G. Zhang · J.J. Tian · J.S. Sun • \\ A.A. Volinsky • L.J. Qiao
}

Received: 25 May 2009 / Accepted: 1 September 2009 / Published online: 24 September 2009

(C) Springer-Verlag 2009

\begin{abstract}
Resonant modes and magnetoelectric performance of layered PZT/Ni and Ni/PZT/Ni cylindrical composites are considered. The first and the second resonant frequencies in the $1-150 \mathrm{kHz}$ range correspond to the axial and the radial modes. Experimental results and theoretical analysis indicate that one should choose the trilayered structure and the first resonant frequency as the working frequency. This study is helpful in design and applications of magnetoelectric devices.
\end{abstract}

PACS 71.20. $\mathrm{Nr} \cdot 71.20 . \mathrm{Be}$

\section{Introduction}

Bulk magnetoelectric (ME) materials can be utilized in devices operating at low frequencies (pico-Tesla sensitivity magnetometer $[1,2]$ ) and at microwave frequencies (electrostatically tunable band-pass filters [3], band-stop filters [4], resonators [5], phase shifters [6]). Most of the devices are based on the layered ME composites, which exhibit stronger $\mathrm{ME}$ effect compared with the bulk particulate ME materials [7].

D.A. Pan · S.G. Zhang ( $\varangle) \cdot$ J.J. Tian $\cdot$ L.J. Qiao

School of Materials Science and Engineering, University of Science and Technology Beijing, Beijing 100083, P.R. China e-mail: zhangshengen@mater.ustb.edu.cn

J.S. Sun

Faculty of Materials and Metallurgical Engineering, Kunming university of Science and Technology, Kunming, Yunnan, 650093, P.R. China

A.A. Volinsky

Department of Mechanical Engineering, University of South

Florida, Tampa, FL 33620, USA
In previous studies plate and cylindrical layered ME composites made by electro-deposition were considered [8-11]. Electrodeposited plate layered ME composites have comparable ME performance to the Terfenol-D/PZT prepared by the gluing method $[8,9]$. Nickel $(\mathrm{Ni}) /$ lead zirconate titanate (PZT)/Ni trilayered composites also have some novel ME properties [10], while cylindrical Ni/PZT bilayered composites exhibit high gain ME effect at resonant frequency under high magnetic field induced by the volume magnetostriction and possibly induction effects [11]. Cylindrical layered ME composites have a significant potential for applications in high magnetic field detection. In this paper, the resonant modes and magnetoelectric performance of the cylindrical magnetoelectric composites with PZT/Ni bilayered and Ni/PZT/Ni trilayered structure are studied.

\section{Experimental}

A hollow $\mathrm{Pb}\left(\mathrm{Zr}_{0.52} \mathrm{Ti}_{0.48}\right) \mathrm{O}_{3}$ (PZT-5H) cylinder (supplied by Institute of Acoustics, Chinese Academy of Sciences) was cut with a height of $8 \mathrm{~mm}$, an inner diameter of $18 \mathrm{~mm}$, and an outer diameter of $20 \mathrm{~mm}$ (1-mm PZT cylinder wall thickness). PZT was polarized at $425 \mathrm{~K}$ in a $50 \mathrm{kV} / \mathrm{cm}$ electric field along the radial directions after being coated with conductive plastic. The poled PZT cylinder was cleaned from the plastic prior to Ni deposition on its surface. Then, the PZT cylinder was bathed in nickel aminosulfonate plating solution to electro-deposit the Ni layer. The composition of the plating solution is described in detail elsewhere [8-11]. For the PZT/Ni bilayered structure, after the cylinder inner wall was protected by the silicone rubber, only the outer surface Ni electrode was deposited. For the $\mathrm{Ni} / \mathrm{PZT} / \mathrm{Ni}$ trilayered structure, $\mathrm{Ni}$ was electro-deposited on 
Fig. 1 A schematic diagram for (a) trilayered and (b) bilayered hollow cylindrical layered composite structures and (c) clamp [10]. Here, $d_{31}$ and $d_{33}$ are the piezoelectric constants of the PZT cylinder
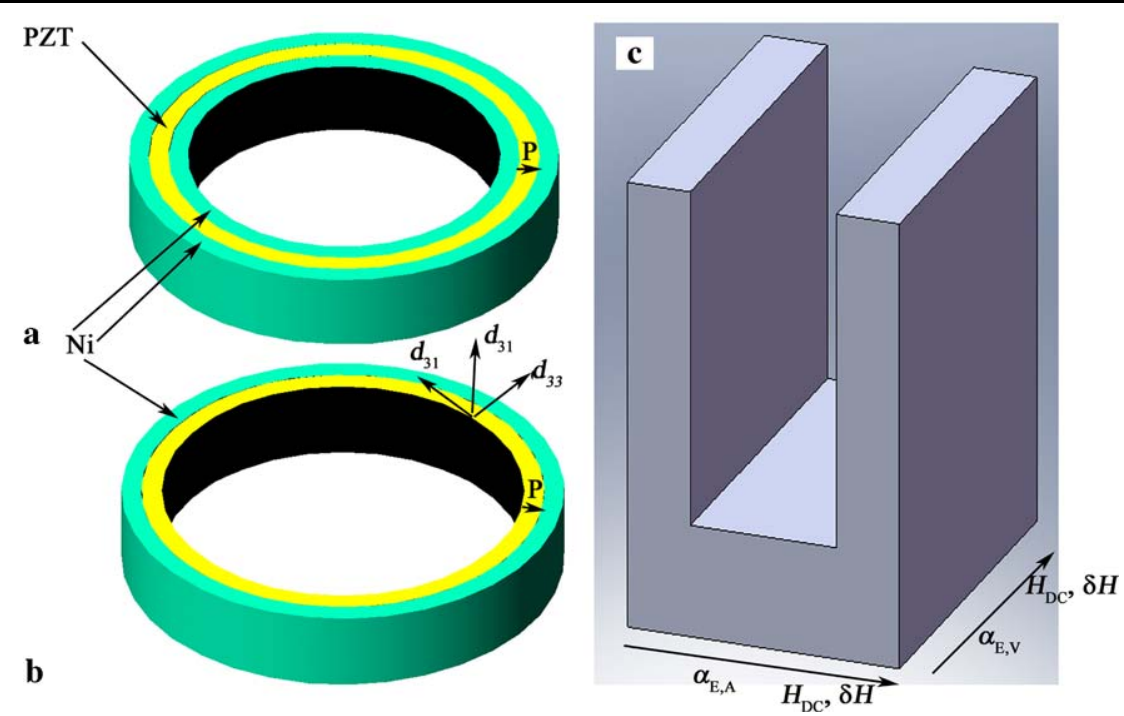

both hollow cylinder sides. During electro-deposition, a cathodic current density of $5 \mathrm{~mA} / \mathrm{cm}^{2}$ was applied to both samples (a cathodic current of $25 \mathrm{~mA}$ and $48 \mathrm{~mA}$ was applied to PZT/Ni and Ni/PZT/Ni structures, respectively) [12]. In order to obtain the same mass of the Ni layers, electrodeposition time of about 100 and 190 hours was used for trilayered and bilayered structures, respectively. The product of cathodic current and electro-deposition time was constant for the two samples. The electro-deposition rates were about $0.01 \mathrm{~mm} / \mathrm{h}$ and $0.005 \mathrm{~mm} / \mathrm{h}$ for trilayered and bilayered structures, respectively. The resulting total Ni thickness was about $1 \mathrm{~mm}$ for both samples, with 1-mm outer $\mathrm{Ni}$ layer for the bilayered sample, and $0.5-\mathrm{mm}$ outer and inner Ni layers for the trilayered sample. Samples are shown schematically in Fig. 1 (a and b).

During the ME measurements, samples were subjected to a bias magnetic field $H_{\mathrm{DC}}$ superimposed with a sinusoidal field $\delta H(1-150 \mathrm{kHz}$ frequency range). The sinusoidal magnetic field with the amplitude $\delta H=22$ Oe was generated by the Helmholtz coils when AC current with the 1-A amplitude was passed through the coils. Voltage, $\delta V$, generated across the PZT cylinder thickness was amplified and measured with an oscilloscope. The ME voltage coefficient was calculated according to $\alpha_{\mathrm{E}}=\delta V /\left(t_{\mathrm{PZT}} \cdot \delta H\right)$, where $t_{\mathrm{PZT}}$ is the PZT thickness, and $\delta H$ is the change in the applied magnetic field. Details about the measurement system have been reported in our previous paper [13]. Each sample was placed in a nylon clamp shown schematically in Fig. 1(c). The groove in the clamp is $8.5-\mathrm{mm}$ wide, $15-\mathrm{mm}$ deep, and 20-mm high. The clamp was fixed at the bottom, and the sample was hanging from its top supported by the voltage measuring wires. Two ME voltage coefficients, $\alpha_{\mathrm{E}, \mathrm{V}}$ and $\alpha_{\mathrm{E}, \mathrm{A}}$, were obtained, corresponding to two conditions, where $H_{\mathrm{DC}}$ and $\delta H$ were applied either along or perpendicular to the groove, as shown in Fig. 1(c).

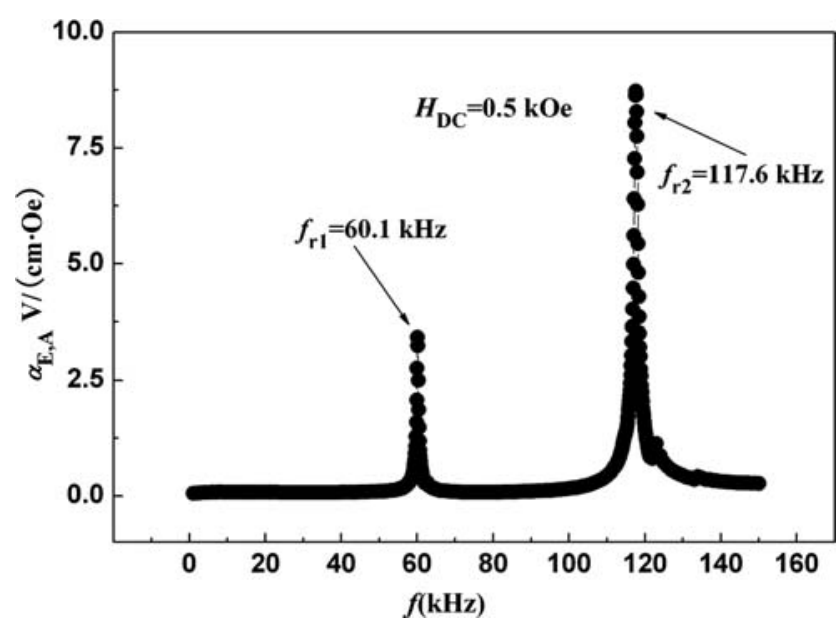

Fig. 2 The dependence of the magnetoelectric voltage coefficient $\alpha_{\mathrm{E}, \mathrm{A}}$ on the applied magnetic field frequency for PZT/Ni bilayered cylindrical ME composites at the applied bias magnetic field $H_{\mathrm{DC}}$ of $0.5 \mathrm{Oe}$. $f_{\mathrm{r} 1}$ and $f_{\mathrm{r} 2}$ are the first and second resonant frequencies

\section{Results and discussion}

The magnetoelectric voltage coefficient $\alpha_{\mathrm{E}, \mathrm{A}}$ dependence on the applied magnetic field frequency for the PZT/Ni bilayered cylindrical ME composite with $H_{\mathrm{DC}}=0.5 \mathrm{kOe}$ is shown in Fig. 2. There are two resonant peaks in the ME voltage coefficient curve as a function of the frequency of the applied magnetic field in the $1-150 \mathrm{kHz}$ range. We denote these frequencies as $f_{\mathrm{r} 1}$ and $f_{\mathrm{r} 2}$, corresponding to the first and the second resonant peaks, respectively. The trilayered structure also exhibits two resonant peaks in the $H_{\mathrm{DC}}$ curve for the same $1-150 \mathrm{kHz}$ frequency range. In this study, we will emphasize the ME effect $\left(\alpha_{\mathrm{E}, \mathrm{A}}\right.$ and $\left.\alpha_{\mathrm{E}, \mathrm{V}}\right)$ dependence on the bias magnetic field $\left(H_{\mathrm{DC}}\right)$ and the frequency of the applied magnetic field $(f)$. 

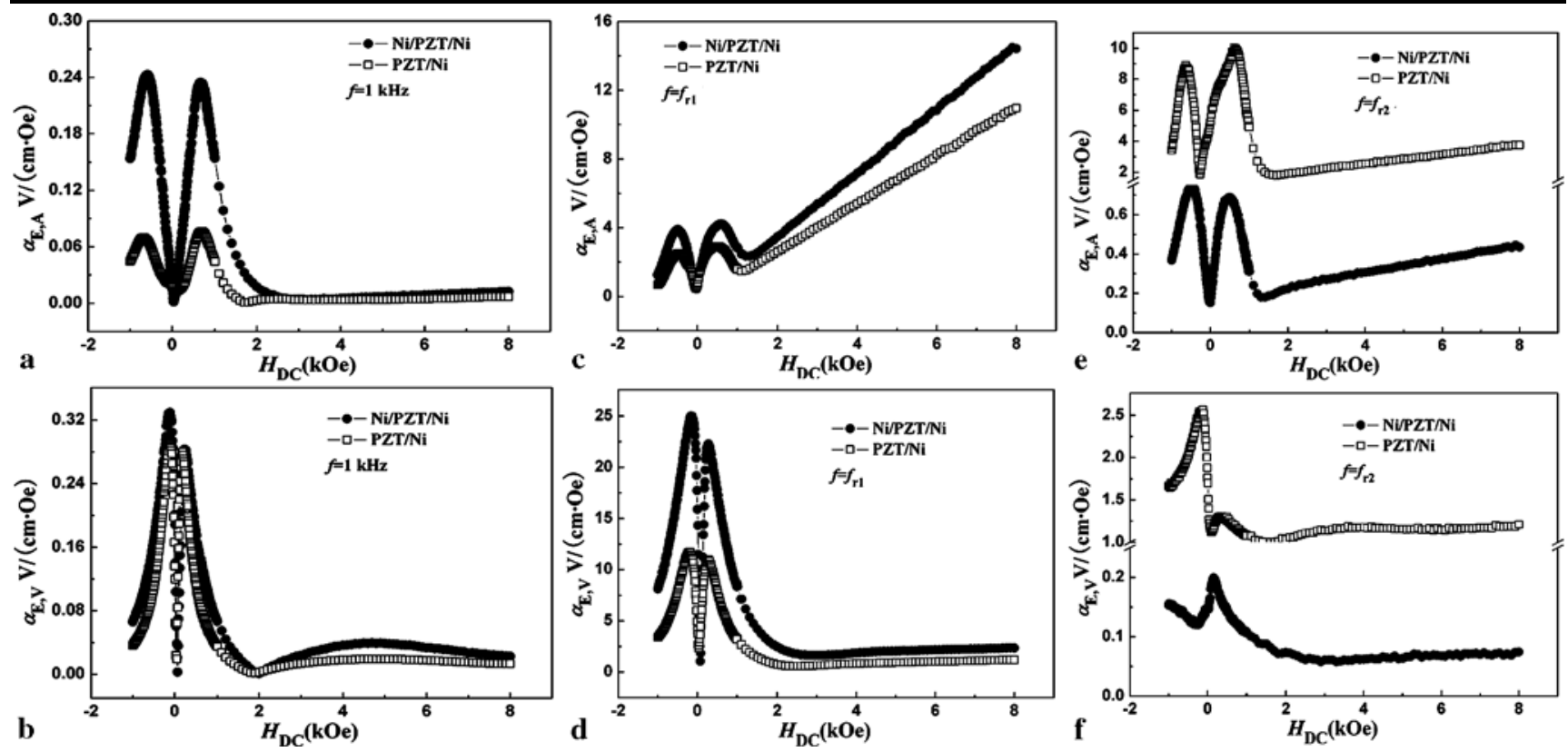

Fig. 3 The dependence of the magnetoelectric voltage coefficients $\alpha_{\mathrm{E}, \mathrm{A}}(\mathbf{a}, \mathbf{c}$, and $\mathbf{e})$ and $\alpha_{\mathrm{E}, \mathrm{V}}(\mathbf{b}, \mathbf{d}$, and $\mathbf{f})$ on the bias magnetic field $H_{\mathrm{DC}}$ varied from -1 to $8 \mathrm{kOe}$ at $1 \mathrm{kHz}$ and on the first resonance frequency

The magnetoelectric voltage coefficients dependence on the bias magnetic field $H_{\mathrm{DC}}$ varied from -1 to $8 \mathrm{kOe}$ at $1 \mathrm{kHz}$ for the $\mathrm{Ni} / \mathrm{PZT} / \mathrm{Ni}$ and PZT/Ni cylindrical composites $\alpha_{\mathrm{E}, \mathrm{A}}$ and $\alpha_{\mathrm{E}, \mathrm{V}}$ is shown in Fig. 3 ( $\mathrm{a}$ and $\mathrm{b}$ ). Figure 3 indicates that $\alpha_{\mathrm{E}, \mathrm{A}}$ and $\alpha_{\mathrm{E}, \mathrm{V}}$ are different for the bilayered and the trilayered structures at $1 \mathrm{kHz}$. The ME voltage coefficients $\alpha_{\mathrm{E}}$ initially increased and then decreased subsequently with $H_{\text {DC }}$ at negative magnetic field when the applied frequency was $1 \mathrm{kHz}$ for bilayered and trilayered samples. With $H_{\mathrm{DC}}$ increasing continuously, the $\alpha_{\mathrm{E}}$ trend for both samples is the same as reported in the previous work [10]. However, there appear two peaks in the $H_{\mathrm{DC}}$ curve in the vertical mode at positive fields.

This effect is induced by the difference in series of infinitesimal plate units and is discussed in the previous work [14]. The cylindrical layered ME composite axial coupling mode can be simplified as a plate trilayered ME composite longitudinal mode (Fig. 4). The dimensions of the simplified plate trilayered ME composite are $h \times L^{\text {eff }} \times t$, where $h$ is the cylinder height, $L^{\text {eff }}$ is the effective length of the simplified plate, and $t$ is the total sample thickness corresponding to the cylinder wall thickness. After simplification, the effective magnetic fields $H^{\text {eff }}$ and $\delta H^{\text {eff }}$ are along the $h$ direction, as shown in Fig. 4. The cylinder in the vertical mode also can be simplified as a plate trilayered ME composite with the effective magnetic fields acting along the thickness $(t)$ and the effective length ( $\left.L^{\text {eff }}\right)$ directions of the plate, simultaneously [14]. In the plate layered ME composites, there was a maximum at low magnetic field $f_{\mathrm{r} 1}$ and the second frequency $f_{\mathrm{r} 2}$ for the Ni/PZT/Ni and PZT/Ni layered cylindrical composites, respectively

of the $H_{\mathrm{DC}}$ curves in $\alpha_{\mathrm{E}, 31}$ (length direction, $L^{\text {eff }}$ ) and $\alpha_{\mathrm{E}, 32}$ (width direction, $h$ ). However, there was a maximum at high magnetic field of the $H_{\mathrm{DC}}$ curves in $\alpha_{\mathrm{E}, 33}$ (thickness direction, $t)[8,15,16]$. That is why there appears one peak in the axial mode but two peaks in the vertical mode at positive fields.

There is a difference between the two samples in the axial mode. That is, at the same bias magnetic field below $2 \mathrm{kOe}$, the $\alpha_{\mathrm{E}}$ of the trilayered structure is larger than that of the bilayered sample, and passing $2 \mathrm{kOe}$, this difference becomes smaller. However, in the vertical mode, the ME voltage coefficients of both samples are similar for the same bias magnetic field below $2 \mathrm{kOe}$, and the $\mathrm{ME}$ effect in the trilayered sample is a little bit larger than in the bilayered sample above $2 \mathrm{kOe}$.

Figure 3(c, d) shows the dependence of the magnetoelectric voltage coefficients $\alpha_{\mathrm{E}, \mathrm{A}}$ and $\alpha_{\mathrm{E}, \mathrm{V}}$ on $H_{\mathrm{DC}}$ varied from $-1 \mathrm{kOe}$ to $8 \mathrm{kOe}$ at $f_{\mathrm{r} 1}$ applied frequency for the $\mathrm{Ni} / \mathrm{PZT} / \mathrm{Ni}$ and PZT/Ni layered cylindrical composites. Figure 3(c) shows that the $\alpha_{\mathrm{E}, \mathrm{A}}$ variation below $1 \mathrm{kOe}$ is the same as in Fig. 3(a) for the two samples. However, with the increasing bias magnetic field, $\alpha_{\mathrm{E}, \mathrm{A}}$ linearly increases. This novel ME effect is caused by the Ni volume magnetostriction under high magnetic field [11].

For ferromagnetic materials, like $\mathrm{Ni}$, the line magnetostriction, $\lambda=\Delta l / l$, increases with bias magnetic field, $H_{\mathrm{DC}}$, and then reaches a saturation value $\lambda_{\mathrm{s}}$ at $H_{\mathrm{s}}$. When $H_{\mathrm{DC}}<H_{\mathrm{S}}$, the volume changes, while the volume magnetostriction $\omega=\Delta V / V$ is too small to be measured [17]. 
Fig. 4 (a) Trilayered cylinder; (b) trilayered plate composite simple bound state applied for the cylindrical layered composite in the axial coupling mode; and (c) in the vertical coupling mode [14]

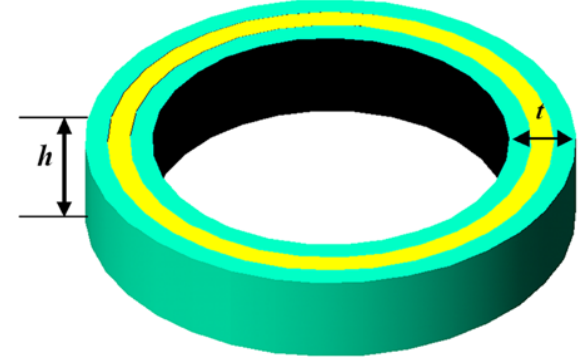

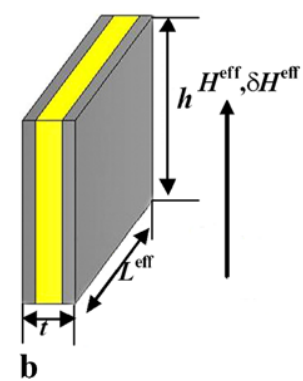

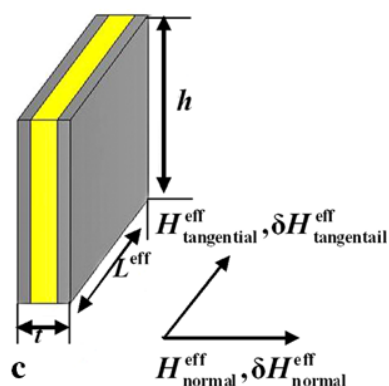

caused by the volume magnetostriction under high magnetic field is weaker than the one caused by the line magnetostriction under low magnetic field. This means that one cannot choose this resonant frequency as the working frequency for the high magnetic field detection devices. Figure 3(f) shows $H_{\mathrm{DC}}$ curve of $\alpha_{\mathrm{E}, \mathrm{V}}$ in the vertical mode. Here, $\alpha_{\mathrm{E}, \mathrm{V}}$ is very weak, and the bilayered sample ME effect is larger than for the trilayered sample. This also indicates that one cannot choose this resonant frequency as the working frequency.

From Fig. 3 it is obvious that the magnetoelectric voltage coefficient of the trilayered sample is larger than that of the bilayered one at low and at the first resonant frequencies. However, at the second resonance frequency, the situation is reversed. This indicates that the first and the second resonant peaks correspond to different resonant modes. In a cylinder, there are axial and radial vibration modes, and thus the first and the second resonant vibrations correspond to the axial and the radial modes, respectively [20]. In the axial resonant mode, the mechanical coupling-induced $\mathrm{ME}$ effect is along the cylinder axis, and the contact area in the trilayered sample is twice larger compared with the bilayered sample. In this mode, the strain transfer in the trilayered sample is more effective than in the bilayered sample, and thus the ME effect induced in the trilayered sample is larger than in the bilayered sample. However, in the radial resonant mode, the main contribution to the $\mathrm{ME}$ effect is along the radial cylinder direction. The Ni cylinder will shrink or expand in the magnetic field, depending in which direction the field is applied. In order to explain the state of stress in the radial mode, we assume that the Ni layer shrinks in the magnetic field and neglect the tangent force in the vertical mode. For the bilayered sample, because of the outer Ni layer shrinking, the PZT hollow cylinder will suffer compressive stress along the radial direction. However, for the trilayered sample, the stress state is more complex than that of the bilayered sample. The middle PZT layer will be under compressive stress in the radial direction induced by the outer $\mathrm{Ni}$ layer shrinking and under tensile stress induced by the inner Ni layer shrinking. The two stresses of the opposite sign act along the radial direction and as a result, the overall radial stress in the PZT is reduced. This is why the magnetoelectric voltage coefficient of bilayered sample is larger than that of 


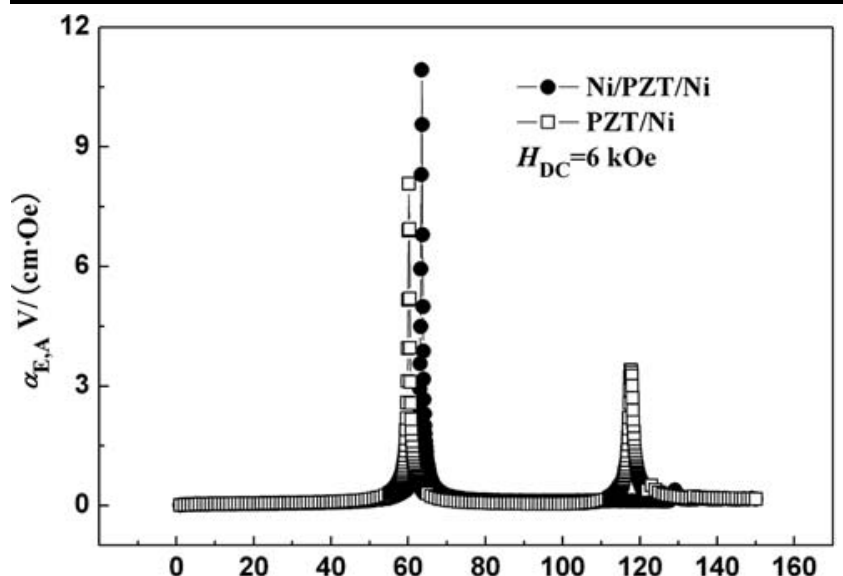

a

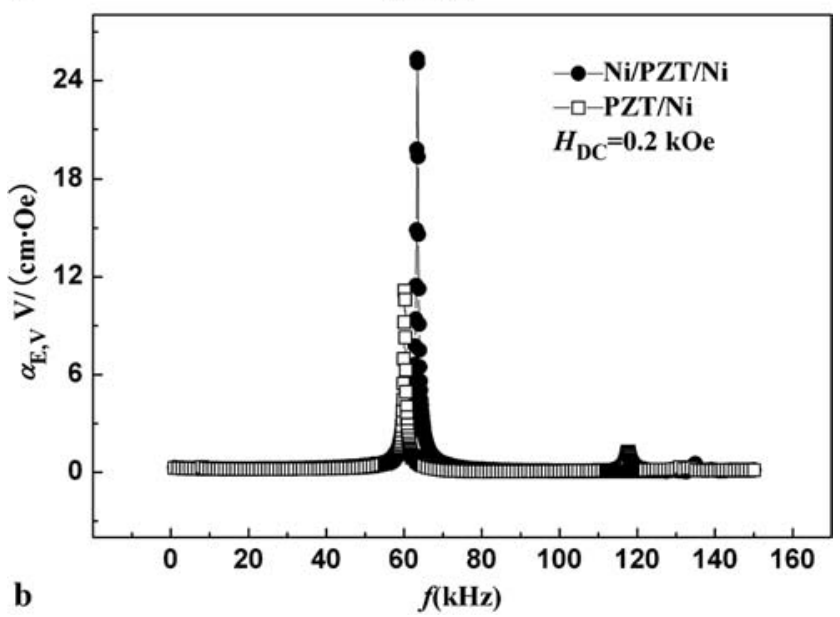

Fig. 5 The dependence of the magnetoelectric voltage coefficients $\alpha_{\mathrm{E}, \mathrm{A}}(\mathbf{a})$ and $\alpha_{\mathrm{E}, \mathrm{V}}(\mathbf{b})$ on the applied magnetic field frequency for $\mathrm{Ni} / \mathrm{PZT} / \mathrm{Ni}$ and PZT/Ni layered cylindrical ME composites when the applied bias magnetic field $H_{\mathrm{DC}}$ is $6 \mathrm{kOe}$ and $0.2 \mathrm{kOe}$, respectively

the trilayered sample as reported above at the second resonant frequency (radial mode).

Comparing Figs. 3(c) and 3(e), the maximum ME voltage coefficient is obtained at high magnetic field of $8 \mathrm{kOe}$ at the first resonant frequency, but at low magnetic field of $0.5 \mathrm{kOe}$ at the second resonant frequency for the bilayered sample. The ME maximum at the first and the second resonant frequencies is almost the same (about $11 \mathrm{~V} /(\mathrm{cm} \cdot \mathrm{Oe})$ ). This indicates that the tangent force is the main cause of stress in the cylinder. That is to say, in the ME effect, the piezoelectric modes of $d_{31}$ in PZT play the main role in the ME effect. Cylindrical PZT ceramic was polarized along the radial direction; the corresponding piezoelectric constants are denoted in Fig. 1(b). The axial and tangential shear stresses are contributing to deformation along $d_{31}$, and the normal compressive/tensile stresses are contributing to deformations along $d_{33}$. Moreover, $d_{33}$ dielectric constant is 3-4 times larger than $d_{31}$.
Figure 5 shows the dependence of the ME voltage coefficients $\alpha_{\mathrm{E}, \mathrm{A}}$ (a) and $\alpha_{\mathrm{E}, \mathrm{V}}$ (b) on the applied magnetic field frequency for $\mathrm{Ni} / \mathrm{PZT} / \mathrm{Ni}$ and $\mathrm{PZT} / \mathrm{Ni}$ layered cylindrical ME composites when $H_{\mathrm{DC}}$ is $0.2 \mathrm{kOe}$ and $6 \mathrm{kOe}$. The trilayered sample has the largest ME voltage coefficient at the first resonant frequency for both $0.2 \mathrm{kOe}$ and $6 \mathrm{kOe} H_{\mathrm{DC}}$. This indicates that one should choose the trilayered structure and the first resonant frequency for the ME device structures as the working frequency, although the ME peak of the bilayered sample is larger than that of the trilayered sample at the second resonant frequency.

\section{Conclusions}

In summary, the first and the second resonant frequencies in the $1-150 \mathrm{kHz}$ range in cylindrical ME composites corresponding to the axial and the radial modes were analyzed. The magnetoelectric voltage coefficient of the trilayered structure is larger than that of the bilayered structure at the first resonant frequency, but this situation is reversed at the second resonant frequency. In order to obtain high performance ME devices, one should choose the trilayered structure and the first resonant frequency as the working frequency.

Acknowledgements This research was supported by the National Natural Science Foundation of China under Grants 50572006, 50802008, and 50874010, by Beijing Natural Science Foundation of China under Grant 2073026, by Program for New Century Excellent Talents in University (NCET) 20060420152, by Scholars and Innovative Research Team in University (IRT 0509), and by the National Natural Science Foundation of China. Alex A. Volinsky would like to acknowledge support from NSF under CMMI-0600266 grant.

\section{References}

1. J. Zhai, Z. Xing, S. Dong, J. Li, D. Viehland, Appl. Phys. Lett. 88, $062510(2006)$

2. J. Zhai, S. Dong, Z. Xing, J. Li, D. Vieland, Appl. Phys. Lett. 91, 123513 (2007)

3. Y.J. Wang, S.W. Or, H.L.W. Chan, X.Y. Zhao, H.S. Luo, Appl. Phys. Lett. 93, 213504 (2008)

4. Y. J Wang, F.F. Wang, S.W. Or, H.L.W. Chan, X.Y. Zhao, H.S. Luo, Appl. Phys. Lett. 93, 113503 (2008)

5. G. Srinivasan, I.V. Zavislyak, A.S. Tatarenko, Appl. Phys. Lett. 89, 152508 (2006)

6. Y.K. Fetisov, G. Srinivasan, Appl. Phys. Lett. 88, 143503 (2006)

7. Q.H. Jiang, Z.J. Shen, J.P. Zhou, Z. Shi, C.W. Nan, J. Eur. Ceram. Soc. 27, 279 (2007)

8. D.A. Pan, Y. Bai, W.Y. Chu, L.J. Qiao, Smart Mater. Struct. 16, $2501(2007)$

9. D.A. Pan, Y. Bai, W.Y. Chu, L.J. Qiao, J. Phys.: Condens. Matter. 20, 025203 (2008)

10. D.A. Pan, Y. Bai, W.Y. Chu, L.J. Qiao, J. Phys. D: Appl. Phys. 41, 022002 (2008)

11. D.A. Pan, Y. Bai, A.A. Volinsky, W.Y. Chu, L.J. Qiao, Appl. Phys. Lett. 92, 052904 (2008) 
12. D.A. Pan, S.G. Zhang, L.J. Qiao, A.A. Volinsky, J. Phys. D: Appl. Phys. 41, 195004 (2008)

13. J. Lu, D.A. Pan, Y. Bai, L.J. Qiao, Meas. Sci. Technol. 19, 045702 (2008)

14. D.A. Pan, S.G. Zhang, A.A. Volinsky, L.J. Qiao, J. Phys. D: Appl. Phys. 41, 205008 (2008)

15. D.A. Pan, S.G. Zhang, A.A. Volinsky, L.J. Qiao, J. Phys. D: Appl. Phys. 41, 172003 (2008)
16. D.A. Pan, J. Lu, Y. Bai, W.Y. Chu, L.J. Qiao, Chin. Sci. Bull. 53, $2124(2008)$

17. F. Richter, U. Lotter, Phys. Status Solidi 34, k149 (1969)

18. C.W. Chen, Magnetism and Metallurgy of Soft Magnetic Materials (North-Holland, Amsterdam, 1977)

19. C.M. Van der Burgt, Philips Res. Rep. 8, 91 (1953)

20. R.D. Blevins, Formulas for Natural Frequency and Mode Shape (Krieger, Melbourne, 1979) 